\title{
Economic and Clinical Burden of Acute Myeloid Leukemia Episodes of Care in the United States: A Retrospective Analysis of a Commercial Payer Database
}

Bhavik J. Pandya, PharmD; Chi-Chang Chen, PhD; Bruno C. Medeiros, MD; Catherine B. McGuiness, MA, MS; Samuel D. Wilson, PhD; Elise Horvath Walsh, MD; and Rolin L. Wade, RPh, MS

\begin{abstract}
BACKGROUND: In the United States, the incidence of acute myeloid leukemia (AML) has steadily increased over the last decade; in 2019, it was estimated that AML would affect 21,450 new patients and lead to 10,920 deaths. Detailed real-world cost estimates and comparisons of key AML treatment episodes, such as in high-intensity chemotherapy (HIC), low-intensity chemotherapy (LIC), hematopoietic stem cell transplantation (HSCT), and relapsed/refractory (R/R), are scarce in the commercially insured U.S. population.
\end{abstract}

OBJECTIVE: To examine health resource utilization (HRU), clinical burden, and direct health care costs across various AML treatment episodes in a large sample of commercially insured U.S. patients.

METHODS: A retrospective cohort analysis was conducted. Patients with newly diagnosed AML were followed to identify the key active treatment episodes across the course of their disease. Data were obtained from 2 sources: IQVIA's Real-World Data (RWD) Adjudicated Claims DatabaseU.S. (formerly known as PharMetrics Plus), which comprises adjudicated claims for more than 150 million unique enrollees across the United States, and IQVIA Charge Detail Master Hospital Database, which has detailed data regarding services received in an inpatient setting. Calculation of all-cause HRU was based on physician office visits, nonphysician office visits, emergency department visits, inpatient visits, and outpatient pharmacy utilization. Calculation of all-cause health care costs was based on total allowed costs and reported by the following cost components: physician office visits, nonphysician office visits, emergency department visits, inpatient visits, and outpatient pharmacy utilization. Symptom and toxicity events were estimated via proxies such as diagnosis codes, procedures, and treatments administered.

RESULTS: The final study sample consisted of 1,542 HIC-induction (HIC-I), 591 HIC-consolidation (HIC-C), 628 LIC, 1,000 patients with HSCT, and 707 patients with R/R AML. Total mean episode costs were highest in R/R episodes $(\$ 439,104)$, followed by HSCT $(\$ 329,621)$, HIC-I $(\$ 198,657)$, HIC-C $(\$ 73,428)$, and LIC $(\$ 53,081)$ episodes. Across all treatment episodes, hospitalization was the largest contributor to cost with mean hospitalization costs ranging from $\$ 308,978$ in the $R / R$ setting to $\$ 49,580$ for patients receiving LIC; of these, costs related to intensive care unit admission were a noteworthy contributor. In patients with R/R AML and HSCT, expenditures related to pharmacy utilization averaged $\$ 24,640$ and $\$ 12,203$, respectively, and expenditures related to physician office visits averaged $\$ 10,926$ and $\$ 6,090$, respectively; these expenditures were much lower across other episodes. Across all categories of symptom and toxicity events, cardiovascular events was the only category of event that was a significant predictor of higher cost across all episodes. Symptom and toxicity events commonly associated with AML were associated with significantly increased costs, especially in R/R episodes.
CONCLUSIONS: This resource utilization and direct health care cost analysis highlights the substantial economic burden associated with key AML treatment episodes in the United States, specifically during HIC-I, HSCT, and $R / R$ episodes.

\section{J Manag Care Spec Pharm. 2020;26(7):849-59}

Copyright $\odot 2020$, Academy of Managed Care Pharmacy. All rights reserved.

\section{What is already known about this subject}

In the United States, the incidence of acute myeloid leukemia (AML) has steadily increased over the past decade.

Analyses examining the costs associated with different AML treatment episodes in the commercially insured U.S. population are rare and difficult to assemble.

\section{What this study adds}

Total mean episode costs were highest in relapsed/refractory episodes $(\$ 439,104)$, followed by hematopoietic stem cell transplantation $(\$ 329,621)$, high-intensity induction chemotherapy $\$ 198,657)$, high-intensity consolidation chemotherapy $(\$ 73,428)$, and low-intensity chemotherapy $(\$ 53,081)$ episodes; inpatient hospitalization was the largest contributor to cost, accounting for roughly $70 \%$ of cost across all treatment groups.

This resource utilization and direct health care cost analysis demonstrate the substantial economic burden associated with various AML treatment episodes, especially during high-intensity induction chemotherapy, hematopoietic stem cell transplantation, and relapsed/refractory episodes.

Hospitalization is a major cost driver across all episodes.

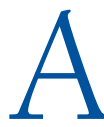
cute myeloid leukemia (AML) is the most common form of acute leukemia in adults in the United States and its incidence has steadily increased over the last decade. In 2019, it was estimated that AML would affect 21,450 new patients and lead to 10,920 deaths. ${ }^{1}$ Outcomes for patients with AML remain unsatisfactory, with a 5 -year overall survival of only $28 \% .^{2}$

While the National Comprehensive Cancer Network guidelines for AML treatment often include chemotherapy with 
cytarabine and anthracyclines and/or hematopoietic stem cell transplantation (HSCT), additional regimens are available, including targeted therapy and immunotherapy. Despite improvements in available treatment options, most patients with AML will relapse, at which point 5-year survival plummets to $5 \%-11 \%{ }^{3,4}$

Detailed real-world cost estimates are important for understanding the economic and physical resources necessary to help facilitate higher standards of care. To date, detailed realworld cost estimates of key AML treatment episodes, such as high-intensity chemotherapy (HIC), low-intensity chemotherapy (LIC), HSCT, and relapsed/refractory (R/R) AML, are scarce and difficult to assemble. To the extent AML-related cost estimates exist, ${ }^{5-8}$ discrepancies in study methodology, therapeutic approaches, and treatment costs make studies hard to interpret and/or not widely applicable. Also, there is limited data regarding the economic and clinical burden associated with the journey from diagnosis to death of a patient with AML, and no studies have used single datasets to understand the continuum of care in AML. As such, this analysis examined health resource utilization (HRU) and direct health care costs, as well as their relationship with clinical outcomes of patients who progress from newly diagnosed to transplant, to relapsed and refractory, and/or to death-all within a single dataset.

\section{Methods}

\section{Study Design}

This retrospective cohort study of health care claims data used IQVIA's Real-World Data (RWD) Adjudicated Claims Database-U.S. (formerly known as PharMetrics Plus) as the primary data source and IQVIA's Charge Detail Master (CDM) as the secondary data source. The study period ran from July 2007 to June 2016; all patients were indexed between January 2008 and March 2016 (Appendix A, available in online article). Index date was defined as the date of the first observed claim with an AML diagnosis. All enrolled patients were newly diagnosed using a 6-month washout period and were required to be continuously enrolled in their health plan for $\geq 6$ months before and $\geq 3$ months after the index date.

\section{Data Sources}

Data from 2 databases were combined and analyzed to achieve study objectives. The primary data source, IQVIA RWD Adjudicated Claims Database, is one of the largest U.S. health plan claims databases, comprising adjudicated claims from more than 150 million unique enrollees. This database has a diverse representation of geography, employers, payers, providers, and therapy areas and is considered representative of the national, commercially insured population in terms of age and gender. As IQVIA owns this database, permission for its use was not necessary. All data are de-identified and fully compliant with the patient confidentiality requirements of the Health
Insurance Portability and Accountability Act (HIPAA). As such, no institutional review board approval was required.

The IQVIA CDM hospital database, the secondary data source, has detailed data on all services received in inpatient settings. Anonymized patient-level data are sourced from hospital charge detail masters within $>350$ short-term, acute-care, and nonfederal hospitals.

The RWD and CDM databases were linked using IQVIA's patented deterministic linking methodology. The RWD database was the primary source used to assess AML burden of illness. Once linked to the RWD, the CDM provided additional details on inpatient use of AML therapies to aid in the identification of patients who received HIC or LIC, as these therapies are frequently administered in the inpatient setting.

\section{Patient Selection}

Patients were required to have a diagnosis of AML in the RWD Adjudicated Claims Database during the sample selection window (January 1, 2008, to March 31, 2016), evidenced by $\geq 2$ nonancillary outpatient claims with an AML diagnosis (International Classification of Diseases, Ninth Revision, Clinical Modification [ICD-9-CM] code 205.00) $>30$ days apart or $\geq 1$ inpatient claim with an AML diagnosis. Ancillary services include laboratory tests, radiology procedures, and other services provided to support the work of the primary physician. Patients were also required to have continuous health plan enrollment in the RWD Adjudicated Claims Database $\geq 6$ months before and $\geq 3$ months after the initial AML diagnosis date. Patients must have had HIC, LIC, or HSCT after the initial AML diagnosis and no observed AML diagnoses $\geq 6$ months before the initial AML diagnosis date to capture patients during the initial diagnosis of AML.

In order to increase the specificity of the study's AML population, patients were excluded if they had a diagnosis of acute lymphoblastic leukemia or a diagnosis of other primary or secondary malignancies (except for myelodysplastic syndrome, chronic myelogenous leukemia, and secondary AML) during the 6-month period before the initial AML diagnosis date. Patients who received primary or secondary best supportive care after the initial AML diagnosis date were included in the original patient pool but excluded from this analysis due to a high degree of heterogeneity (precluding the ability to make valid inferences).

\section{Treatment Definitions}

High-intensity induction chemotherapy (HIC-I) was defined as evidence of inpatient high-dose cytarabine plus anthracycline use within 3 months of diagnosis. The HIC-I episodes began with the first observed HIC (inpatient or outpatient) and ended with initiation of another active treatment (e.g., postremission chemotherapy or HSCT), including consolidation. In patients without a subsequent treatment regimen, the HIC-I episode ended 30 days after HIC-I treatment or at the end of observed follow-up (whichever occurred first). 
High-intensity consolidation chemotherapy (HIC-C) was defined as evidence of cytarabine plus anthracycline use during the 2 months following HIC. The HIC-C episodes began with the first of up to 4 cycles of cytarabine (administered in the outpatient setting or inpatient setting if the length of stay was $\leq 7$ days) given after HIC induction and ended with initiation of another active treatment. In patients without a subsequent treatment regimen, the HIC consolidation episode ended 30 days after completion of consolidation treatment (up to 4 cycles) or at the end of observed follow-up (whichever occurred first).

LIC was defined as evidence of low-intensity cytarabine, anthracycline, 5-azacytidine, decitabine, clofarabine, or gemtuzumab ozogamicin in the outpatient setting within 3 months of the initial AML diagnosis. Episodes began with receipt of the first LIC agent (within 90 days postindex) and ended with the initiation of another active treatment. In patients without a subsequent treatment regimen, LIC episodes ended 30 days after LIC completion, with a diagnosis code indicative of relapsed disease, the initiation of supportive care, or end of follow-up (whichever occurred first). The HIC and LIC cohorts were mutually exclusive.

The HSCT episodes were defined by a transplant-specific diagnosis or procedure code. The HSCT episodes were defined starting 60 days before the observed transplant procedure date and ending with the initiation of another active treatment, with a diagnosis code indicative of relapsed disease, initiation of secondary supportive care, or end of follow-up (whichever occurred first). In this study, HSCT procedures were not divided into autologous or allogenic procedures.

The R/R subgroup was defined by either an ICD-9/10-CM diagnosis code for relapsed AML (205.02) or the observation of a new line of therapy (LOT) after the initial therapy, implying failure of the first LOT. A new LOT was defined as initiation of treatment following a 90-day chemotherapy-free period after last observed chemotherapy of interest (representing relapsed AML) or upon initiation of treatment with any new chemotherapy agent following completion of the first 60 days of HIC or LIC treatment (representing primary refractory AML).

In cases where both the diagnosis code and a second LOT were observed, disease was considered R/R, and the earlier event defined the R/R episode start date. The R/R episode was validated by a detailed examination of observed LOTs (i.e., specific chemotherapy drugs received, temporal relationship between LOTs [onset/offset timing and the criteria used to determine each], HSCT, and relapsed ICD codes) guided by expert clinical oncologists. Patients indexed to the relapsed disease code were required to have evidence of treatment (chemotherapy or HSCT) within 30 days after the R/R episode start date to validate occurrence of relapsed disease. It is important to note that in the current study, an emergency department (ED) visit that led to subsequent inpatient care was categorized as part of that inpatient visit.

\section{Endpoints}

Demographic characteristics were measured at the time of the initial AML diagnosis and included age, sex, U.S. geographic region, and payer type. Disease characteristics were measured over the 6 months before the initial AML diagnosis date. Diagnosis codes were used to identify general comorbid conditions of interest including cardiovascular, metabolic, renal, hepatic, pulmonary, musculoskeletal, and nervous system illnesses. Charlson Comorbidity Index (CCI) scores were calculated excluding AML diagnoses.

All-cause HRU was reported for the following resource categories: physician office visits, nonphysician office visits (e.g., laboratory claim, ancillary care visit), ED visits, inpatient visits, and pharmacy utilization (outpatient). All-cause health care costs were reported as total health plan allowed costs incurred (i.e., all claims observed during episodes that included, but were not limited to, the above resource categories), in addition to the following cost components: physician office visits, nonphysician office visits, ED visits, inpatient visits, and outpatient pharmacy utilization.

Health care resource utilization and costs were reported for each episode of care, per episode start and end definitions. Category costs were reported for the total cohort (i.e., mean inpatient costs were reported for the total R/R episode, regardless of whether every patient with R/R incurred inpatient costs). Costs were adjusted to 2016 U.S. dollars using the medical care component of the Consumer Price Index for All Urban Consumers.

Symptom and toxicity events (SxTox) measured during episodes were identified via diagnostic/treatment codes and reported as frequencies for blood and lymphatic system disorders, gastrointestinal (GI) disorders, bleeding events, infections and infestations, nervous system disorders, skin and subcutaneous tissue disorders, vascular disorders, renal disorders, liver disorders, and cardiovascular disorders (Appendix B, available in online article).

\section{Statistical Analyses}

All analyses used SAS version 9.3 (SAS Institute, Cary, NC). Descriptive analyses were conducted, and generalized linear models (GLMs) with a gamma distribution and log link function (selected based on the tendency for skewness of health care cost data) were used to assess the contribution of SxTox to total episode costs while controlling for age, gender, and CCI score. The dependent variable was total episode cost, and the independent variables included age, gender, CCI, and the binary SxTox categories (blood and lymphatic system disorders, GI disorders, bleeding events, infections and infestations, nervous system disorders, skin and subcutaneous tissue disorders, vascular disorders, renal disorders, liver disorders, and cardiovascular disorders) where the reference groups were patients without a given disorder. 
TABLE 1

Baseline Patient Characteristics

\begin{tabular}{|c|c|c|c|c|c|}
\hline & \multicolumn{2}{|r|}{ HIC } & \multirow[b]{2}{*}{ LIC } & \multirow[b]{2}{*}{ HSCT } & \multirow[b]{2}{*}{$\mathrm{R} / \mathrm{R}$} \\
\hline & Induction & Consolidation & & & \\
\hline & $(\mathrm{n}=1,542)$ & $(\mathrm{n}=591)$ & $(n=628)$ & $(\mathrm{n}=1,000)$ & $(\mathrm{n}=707)$ \\
\hline \multicolumn{6}{|l|}{ Age (years) } \\
\hline Mean & 47.0 & 47.0 & 64.9 & 51.4 & 52.0 \\
\hline SD & 11.3 & 10.6 & 13.4 & 12.0 & 12.5 \\
\hline Median & 50.0 & 50.0 & 67.0 & 54.0 & 54.0 \\
\hline \multicolumn{6}{|l|}{ Gender, (\%) } \\
\hline Male & 51.8 & 53.0 & 59.7 & 55.4 & 53.3 \\
\hline Female & 48.2 & 47.0 & 40.3 & 44.6 & 46.7 \\
\hline \multicolumn{6}{|c|}{ Geographic region, (\%) } \\
\hline Northeast & 21.1 & 22.7 & 19.9 & 20.6 & 24.8 \\
\hline Midwest & 31.4 & 29.4 & 30.1 & 31.2 & 29.8 \\
\hline South & 39.0 & 40.1 & 32.0 & 40.0 & 37.3 \\
\hline West & 8.5 & 7.8 & 18.0 & 8.2 & 8.1 \\
\hline \multicolumn{6}{|l|}{ Payer type, (\%) } \\
\hline Commercial & 65.0 & 63.5 & 55.1 & 64.7 & 62.2 \\
\hline Self-insured & 32.9 & 34.2 & 33.0 & 33.4 & 34.7 \\
\hline Medicare risk & 0.0 & 0.0 & 5.6 & 0.5 & 0.7 \\
\hline Medicaid & 1.7 & 1.9 & 1.6 & 0.8 & 1.6 \\
\hline Unknown & 0.5 & 0.5 & 4.8 & 0.6 & 0.8 \\
\hline \multicolumn{6}{|l|}{ Health plan type, (\%) } \\
\hline $\mathrm{HMO}$ & 8.5 & 8.3 & 12.3 & 9.4 & 10.3 \\
\hline $\mathrm{PPO}$ & 81.3 & 81.6 & 72.6 & 82.4 & 79.5 \\
\hline POS & 5.3 & 4.7 & 3.3 & 3.6 & 3.8 \\
\hline Consumer-directed & 0.9 & 1.5 & 0.5 & 0.4 & 1.3 \\
\hline Indemnity & 3.4 & 3.6 & 10.8 & 3.7 & 4.4 \\
\hline All other & 0.5 & 0.3 & 0.5 & 0.5 & 0.7 \\
\hline \multicolumn{6}{|c|}{$\begin{array}{l}\mathrm{HIC}=\text { high-intensity chemotherapy; } H M O=\text { health maintenance organization; } \\
H S C T=\text { hematopoietic stem cell transplantation; } L I C=\text { low-intensity chemotherapy; } \\
P O S=\text { point of service; } P P O=\text { preferred provider organization; } R / R=\text { relapsed } \\
\text { refractory; } S D=\text { standard deviation. }\end{array}$} \\
\hline
\end{tabular}

Bleeding events and infections were excluded from the final model as they were correlated with blood and lymphatic system disorders. Results of independent variable parameter estimates were reported as exponentiated values with corresponding 95\% confidence intervals to provide the relative contribution to costs associated with SxTox of interest.

\section{Results}

\section{Patient Sample and Baseline Characteristics}

A total of 21,994 patients with $\geq 2$ outpatient claims with an AML diagnosis $>30$ days apart or one inpatient claim with an AML diagnosis were identified. After applying all inclusion criteria, 7,883 patients with AML remained in the sample. Of the 7,883 patients with AML, 1,542 (19.6\%) patients were further identified as having an HIC-I episode; 591 (7.5\%) with an HIC-C episode; 628 (8.0\%) with an LIC episode; 1,000 (12.7\%) with an HSCT episode; and 707 (9\%) with an R/R episode of care. Patients with AML who were not classified as HIC, LIC, HSCT, or R/R could have undergone HIC, LIC, and/or HSCT but may have failed to meet specific criteria used to define the treatment episode due to data incompleteness.

Patient demographics and baseline characteristics can be seen in Table 1. Patient ages were similar across groups (range $=47.0-52.0$ years) except for patients with an LIC episode (mean $=64.9$ years). The proportion of males were similar across each group, ranging from $51.8 \%$ in the HIC-I episode group to $59.7 \%$ in the LIC episode group. Patients were most often located in the southern United States, and the majority were commercially insured and participated in a preferred provider organization plan. The most frequently observed comorbid conditions were hypertension (34.9\%), dyslipidemia (29.4\%), and osteoarthritis (25.4\%).

The duration (mean \pm standard deviation $[\mathrm{SD}]$ ) of each episode of care was longest in patients with R/R AML (14.77 [15.21] months), followed by patients with HSCT (6.35 [9.77] months), the HIC-I cohort (2.09 [0.37] months), the LIC cohort (1.99 [0.99] months), and the HIC-C cohort (1.52 [1.04] months). The mean (SD) length of hospital stay was longest in the HIC-I sample (26 [13] days), followed by the HSCT (19 [14] days), R/R (174 [32] days), and HIC-C (7 [4] days) samples. The mean (SD) number of total days in the hospital was largest in the R/R sample (75 [394] days), followed by the HIC-I (44 [22] days), HSCT (38 [32] days), and HIC-C (16 [13] days) samples. The majority of patients had at least one outpatient physician office visit (range $=93.7 \%-99.0 \%$ ).

\section{Health Care Resource Cost and Utilization}

Total mean (SD) episode costs were highest in the R/R episodes $(\$ 439,104$ [ $\$ 405,475]$; note: $67.3 \%$ of patients with R/R had HSCT), followed by HSCT $(\$ 329,621[\$ 288,707])$, HIC-I $(\$ 198,657[\$ 190,751])$, HIC-C $(\$ 73,428[\$ 72,811])$, and LIC $(\$ 53,081[\$ 56,715]$ ) episodes (Table 2). Hospitalization was the largest contributor to cost, accounting for roughly $70 \%$ of costs across all treatment groups. Within hospitalization, intensive care unit (ICU) mean (SD) admission costs were a noteworthy contributor, ranging from $\$ 307,446(\$ 291,748)$ in HSCT to $\$ 52,727(\$ 87,926)$ for LIC among patients who were admitted to the ICU. For R/R, the majority of costs (70\%) were associated with hospitalization (mean $\pm \mathrm{SD}=\$ 308,978$ [\$306,987]), followed by nonphysician office visits $(\$ 30,909[\$ 49,315])$ and outpatient pharmacy costs $(\$ 24,640[\$ 46,275])$. Similarly, for HSCT, the majority of the costs (79\%) were associated with hospitalization $(\$ 259,873[\$ 247,198])$, followed by nonphysician office visits $(\$ 20,746[\$ 47,692])$ and outpatient pharmacy costs $(\$ 12,203[\$ 26,716])$. Unlike other treatment episodes, HIC-I episodes required hospitalization in all (100\%) patients, which accounted for the majority of the HIC-I episode costs $(\$ 179,590$ [\$187,478]), with $\$ 2,972(\$ 3,868)$ attributed to physicians' office visits and $\$ 3,186(\$ 4,715)$ attributed to outpatient pharmacy. The HIC-C episodes were also likely to require hospitalization (98.1\%; Table 3), but the associated costs (SD) were relatively low $(\$ 56,645[\$ 67,388])$ compared 


\section{Economic and Clinical Burden of Acute Myeloid Leukemia Episodes of Care in the United States: A Retrospective Analysis of a Commercial Payer Database}

TABLE 2

All-Cause Direct Health Care Costs

\begin{tabular}{|c|c|c|c|c|c|}
\hline & \multicolumn{2}{|c|}{ HIC } & \multirow{2}{*}{$\begin{array}{c}\text { LIC } \\
(n=628) \$\end{array}$} & \multirow{2}{*}{$\begin{array}{c}\text { HSCT } \\
(\mathrm{n}=1,000) \$\end{array}$} & \multirow{2}{*}{$\begin{array}{c}\mathrm{R} / \mathrm{R} \\
(\mathrm{n}=707) \$\end{array}$} \\
\hline & $\begin{array}{l}\text { Induction } \\
(\mathrm{n}=1,542) \$\end{array}$ & $\begin{array}{l}\text { Consolidation } \\
(\mathbf{n}=591) \$\end{array}$ & & & \\
\hline \multicolumn{6}{|l|}{ Total costs } \\
\hline Mean & 198,657 & 73,428 & 53,081 & 329,620 & 439,104 \\
\hline SD & 190,751 & 72,811 & 56,715 & 288,707 & 405,475 \\
\hline Median & 155,485 & 55,160 & 36,659 & 252,245 & 340,862 \\
\hline \multicolumn{6}{|l|}{ Physician office visits } \\
\hline Incidence of $\geq 1$ visit, $\mathrm{n}(\%)$ & $1,482(96.1)$ & $554(93.7)$ & $613(97.6)$ & $990(99.0)$ & $690(97.6)$ \\
\hline Mean & 2,972 & 1,090 & 1,531 & 6,090 & 10,926 \\
\hline SD & 3,868 & 1,259 & 2,416 & 12,869 & 13,645 \\
\hline Median & 1,345 & 668 & 832 & 3,196 & 7,215 \\
\hline \multicolumn{6}{|l|}{ Emergency visits } \\
\hline Incidence of $\geq 1$ visit, $\mathrm{n}(\%)$ & $441(28.6)$ & $154(26.1)$ & $174(27.7)$ & $269(26.9)$ & $385(54.5)$ \\
\hline Mean & 1,179 & 1,043 & 1,235 & 3,956 & 4,301 \\
\hline SD & 4,641 & 2,660 & 2,065 & 21,005 & 20,941 \\
\hline Median & 390 & 344 & 586 & 706 & 861 \\
\hline \multicolumn{6}{|l|}{ Inpatient visits } \\
\hline Incidence of $\geq 1$ visit, $\mathrm{n}(\%)$ & $1,542(100.0)$ & $580(98.1)$ & $225(35.8)$ & $949(94.9)$ & $664(93.9)$ \\
\hline Mean & 179,590 & 56,645 & 49,580 & 259,873 & 308,978 \\
\hline SD & 187,478 & 67,388 & 61,154 & 247,198 & 306,987 \\
\hline Median & 135,942 & 38,831 & 27,914 & 199,765 & 228,916 \\
\hline \multicolumn{6}{|c|}{ Outpatient pharmacy utilization } \\
\hline Incidence of $\geq 1$ visit, $\mathrm{n}(\%)$ & $1,389(90.1)$ & $545(92.2)$ & $562(89.5)$ & $936(93.6)$ & $637(90.1)$ \\
\hline Mean & 3,186 & 2,465 & 2,895 & 12,203 & 24,640 \\
\hline SD & 4,715 & 4,156 & 5,226 & 26,716 & 46,275 \\
\hline Median & 1,044 & 571 & 744 & 4,579 & 11,548 \\
\hline
\end{tabular}

with HSCT, R/R, and HIC-I episodes. While patients with LIC had the lowest hospitalization rate (35.8\%), these made up the largest component of total episode costs $(\$ 49,580[\$ 61,154])$.

\section{Effect of SxTox on Episode Costs and Their Rate of Occurrence}

Patients discussed in this study experienced a wide range of SxTox, some of which made a noteworthy effect on episode costs (Table 4). After adjusting for age, gender, and CCI score, multivariate GLM results revealed cardiovascular SxTox were significant predictors of total episode costs among patients with HIC-I, HIC-C, LIC, and R/R episodes: HIC-I (22\% higher; $P<0.001$ ), HIC-C (23\% higher; $P=0.005)$, LIC (36\% higher; $P<0.001$ ), and $R / R$ code episodes (26\% higher; $P=0.001$ ). The remaining SxTox of interest (blood and lymphatic system disorders, GI disorders, liver disorders, nervous system disorders, renal disorders, skin and subcutaneous tissue disorders, and vascular disorders) had varying degrees of effect depending on the episode type. SxTox events had the greatest effect on total costs of R/R episodes, with significantly increased costs $(P \leq 0.05)$, relative to patients without SxTox, observed in all 8 SxTox categories of interest.

The most frequently observed categories of interest were blood and lymphatic system disorders and infections and infestations (Table 5). The patients receiving HIC-I experienced blood and lymphatic system disorders (98.8\%), infections and infestations (91.1\%), GI disorders (69.1\%), and nervous system disorders (56.2\%). The patients receiving HIC-C experienced blood and lymphatic system disorders (96.4\%), infections and infestations (89.5\%), and bleeding events (73.6\%). The patients receiving LIC experienced blood and lymphatic system disorders (84.1\%), infections and infestations (64.3\%), and bleeding (54.0\%). Patients who received HSCT experienced blood and lymphatic system disorders (96.6\%), infections and infestations (95.7\%), GI disorders (81.5\%), bleeding events (60.0\%), and nervous system disorders (54.4\%). Patients with R/R AML experienced blood and lymphatic system disorders (97.2\%), infections and infestations (97.3\%), GI disorders (86.6\%), bleeding $(72.0 \%)$, cardiovascular disorders (69.6\%), and nervous system disorders (65.8\%). It is worth noting that patients in the HIC-I, HSCT, and R/R cohorts appeared to experience SxTox of interest at higher frequencies compared with patients in the HIC-C and LIC cohorts.

\section{Discussion}

Between 2017 and 2019, a number of therapies have come to the U.S. market, including enasidenib (IDH2-mutated R/R AML), 


\section{Economic and Clinical Burden of Acute Myeloid Leukemia Episodes of Care in the United States: A Retrospective Analysis of a Commercial Payer Database}

TABLE 3 All-Cause Health Care Resource Utilization During Episodes of Care

\begin{tabular}{|c|c|c|c|c|c|}
\hline & \multicolumn{2}{|c|}{ HIC } & \multirow{2}{*}{$\begin{array}{c}\text { LIC } \\
(n=628)\end{array}$} & \multirow{2}{*}{$\begin{array}{c}\text { HSCT } \\
(n=1,000)\end{array}$} & \multirow{2}{*}{$\begin{array}{c}\mathrm{R} / \mathrm{R} \\
(\mathrm{n}=707)\end{array}$} \\
\hline & $\begin{array}{l}\text { Induction } \\
(\mathrm{n}=1,542)\end{array}$ & $\begin{array}{l}\text { Consolidation } \\
\quad(\mathrm{n}=591)\end{array}$ & & & \\
\hline \multicolumn{6}{|l|}{ Duration of episode, months } \\
\hline Mean & 2.1 & 1.5 & 2.0 & 6.4 & 14.8 \\
\hline SD & 0.4 & 1.0 & 1.0 & 9.8 & 15.2 \\
\hline Median & 2.0 & 1.2 & 2.0 & 2.9 & 9.9 \\
\hline \multicolumn{6}{|l|}{ Physician office visits } \\
\hline Incidence of $\geq 1$ visit, $\mathrm{n}(\%)$ & $1,482(96.1)$ & $554(93.7)$ & $613(97.6)$ & $990(99.0)$ & $690(97.6)$ \\
\hline \multicolumn{6}{|c|}{ Number of unique visits in all patients } \\
\hline Mean & 22.9 & 8.8 & 13.0 & 39.2 & 76.8 \\
\hline SD & 28.5 & 8.7 & 13.6 & 42.8 & 71.2 \\
\hline Median & 10.0 & 6.0 & 9.0 & 24.5 & 58.0 \\
\hline \multicolumn{6}{|l|}{ Emergency department visits } \\
\hline Incidence of $\geq 1$ visit, n (\%) & $441(28.6)$ & $154(26.1)$ & $174(27.7)$ & $269(26.9)$ & $385(54.5)$ \\
\hline \multicolumn{6}{|c|}{ Number of unique visits in all patients } \\
\hline Mean & 1.3 & 1.4 & 1.6 & 2.7 & 3.4 \\
\hline SD & 1.5 & 1.5 & 1.4 & 7.7 & 10.0 \\
\hline Median & 1.0 & 1.0 & 1.0 & 1.0 & 2.0 \\
\hline \multicolumn{6}{|l|}{ Inpatient visits } \\
\hline Incidence of $\geq 1$ visit, $\mathrm{n}(\%)$ & $1,542(100)$ & $580(98.1)$ & $225(35.8)$ & $949(94.9)$ & $664(93.9)$ \\
\hline \multicolumn{6}{|c|}{ Number of unique visits in all patients } \\
\hline Mean & 1.9 & 2.2 & 1.4 & 2.3 & 4.5 \\
\hline SD & 0.8 & 1.2 & 0.8 & 1.8 & 3.9 \\
\hline Median & 2.0 & 2.0 & 1.0 & 2.0 & 3.0 \\
\hline \multicolumn{6}{|c|}{ Outpatient pharmacy utilization } \\
\hline Incidence of $\geq 1$ visit, $\mathrm{n}(\%)$ & $1,389(90.1)$ & $545(92.2)$ & $562(89.5)$ & $936(93.6)$ & $637(90.1)$ \\
\hline \multicolumn{6}{|c|}{ Number of unique visits in all patients } \\
\hline Mean & 9.1 & 8.3 & 11.0 & 37.5 & 83.1 \\
\hline $\mathrm{SD}$ & 5.2 & 6.1 & 8.8 & 62.1 & 99.1 \\
\hline Median & 8.0 & 7.0 & 9.0 & 19.0 & 50.0 \\
\hline
\end{tabular}

midostaurin, gemtuzumab ozogamicin, venetoclax (1L-unfit AML), ivosidenib (IDH1-mutated R/R AML), glasdegib, and gilteritinib (FLT3-mutated R/R AML), with several additional agents in the late stages of investigation (e.g., quizartinib and crenolanib). These therapies may help improve prognosis of patients with AML, especially R/R AML, thanks to varied mechanisms of action and/or targets. As data for this analysis were collected before approval for these new therapies, this study serves as a baseline for the clinical and economic burden associated with AML, allowing for comparisons related to the cost-benefit of emerging treatments.

The data show HRU, cost, and clinical burden varied depending on AML episode type. Specifically, R/R, HSCT, and HIC-I AML episodes were associated with the highest resource, economic, and SxTox-related burden. These findings are similar to other work done in this area, ${ }^{5,9,10}$ where the majority of costs for AML episodes appeared to be driven by inpatient hospitalization (average of roughly $70 \%$ of cost, across all episodes), relative to other common cost-accruing events such as outpatient office visits and pharmacy costs (each average $>7 \%$ of cost, across all episodes). This suggests opportunities to avoid inpatient care, in favor of outpatient care, should be used.

Not surprisingly, SxTox during treatment were associated with significant clinical and economic burden, especially during R/R episodes where all 8 categories of SxTox (cardiovascular disorders, blood and lymphatic system disorders, GI disorders, liver disorders, nervous system disorders, renal disorders, skin and subcutaneous tissue disorders, and vascular disorders) contributed to higher costs. Cardiovascular disorders consistently increased total costs across episodes: HIC-I (22\% higher; $P<0.001)$, HIC-C (23\% higher; $P=0.005)$, LIC (36\% higher; $P<0.001$ ), and R/R code episodes (26\% higher; $P<0.001)$; this may be due to the inherently expensive nature of testing and treating cardiovascular disorders as well as the link between AML and cardiovascular disorders. ${ }^{11}$

While dollar-for-dollar comparisons are difficult to make, the findings discussed here are generally consistent with previous studies. Preussler et al. (2017) reported mean adjusted 


\section{Economic and Clinical Burden of Acute Myeloid Leukemia Episodes of Care in the United States: A Retrospective Analysis of a Commercial Payer Database}

TABLE 5 Occurrence of Symptom and Toxicity Events During Each Episode ${ }^{a}$

\begin{tabular}{|c|c|c|c|c|c|}
\hline & HIC-Induction & HIC-Consolidation & LIC & HSCT & $\mathrm{R} / \mathrm{R}$ \\
\hline SxTox of Interest & $(\mathrm{n}=1,542)$ & $(\mathrm{n}=591)$ & $(n=628)$ & $(\mathrm{n}=1,000)$ & $(\mathrm{n}=707)$ \\
\hline Blood and lymphatic system disorders & 98.8 & 96.4 & 84.1 & 96.6 & 97.2 \\
\hline Infections and infestations & 91.1 & 89.5 & 64.3 & 95.7 & 97.3 \\
\hline Gastrointestinal disorders & 69.1 & 48.6 & 47.5 & 81.5 & 86.6 \\
\hline Bleeding events & 39.0 & 73.6 & 54.0 & 60.0 & 72.0 \\
\hline Nervous system disorders & 56.2 & 36.4 & 17.4 & 54.4 & 65.8 \\
\hline Cardiovascular disorders & 44.6 & 27.7 & 36.5 & 52.8 & 69.6 \\
\hline Skin and subcutaneous tissue disorders & 31.2 & 9.1 & 6.1 & 33.1 & 41.7 \\
\hline Vascular disorders & 14.2 & 9.1 & 7.0 & 17.4 & 33.7 \\
\hline Renal disorders & 17.8 & 11.0 & 27.9 & 26.6 & 37.5 \\
\hline Liver disorders & 6.7 & 3.0 & 4.0 & 9.4 & 16.8 \\
\hline
\end{tabular}

accurrence reported as percentage.

HIC = high-intensity chemotherapy; HSCT = hematopoietic stem cell transplantation; LIC=low-intensity chemotherapy; R/R=relapsed/refractory; SxTox =symptom and toxicity events.

in this analysis. Capturing the data for best supportive care separately with this methodology is difficult due to the barriers in defining the beginning and end of such an episode. Patients who receive active therapy typically also receive best supportive care. Therefore, a retrospective claims database is not a reliable means to gain this information.

It is also possible that not all study patients receiving active treatments, such as HIC/LIC, were captured in this study. In the current study for example, patients with AML who were not classified as HIC, LIC, HSCT, or R/R could have undergone HIC, LIC, and/or HSCT but may have failed to meet specific criteria used to define the treatment episode due to data incompleteness. Furthermore, the 3-month postindex requirement may also have introduced a mortality bias in the population as patients who lost enrollment due to death within 3 months of index were excluded from the final study sample, thereby yielding a final study sample with different underlying characteristics when compared with the general population.

The results from the current study may be limited by coding errors made by providers. As the study sample was anonymized to comply with HIPAA regulations, such errors cannot be identified or corrected. As such, these results should be interpreted in the context of the current study sample. Nevertheless, any resulting bias due to coding errors is likely to be random in nature, affecting all patients to an equal degree.

Finally, as the study sample was principally composed of commercially insured patients, the findings are not necessarily generalizable to the uninsured, Medicare fee-for-service, or Medicaid populations. This is especially relevant in AML, considering the median age of the disease; younger, commercially insured patients represented in this sample may include more patients with HSCT, compared with older patients with less commercial coverage.

\section{Conclusions}

This study constitutes the largest retrospective RWD analysis of the economic and clinical burden of AML episodes of care in the United States before approval of targeted agents. Our findings suggest AML episodes are characterized by varying, yet substantial, levels of economic burden. AML is also characterized by substantial physical burden due to disease- and treatment-related SxTox that are responsible for increases to the total cost of care. This, paired with the fact that patients who are diagnosed with AML have poor survival rates, suggests there is a need for therapies with lower toxicity and better efficacy. Emerging treatments that draw on the genetic underpinnings of AML subtypes may help address these needs.

\section{Authors}

BHAVIK J. PANDYA, PharmD; SAMUEL D. WILSON, PhD; and ELISE HORVATH WALSH, MD, Health Economics \& Outcomes Research - Oncology, Medical Affairs Americas, Astellas Pharma U.S., Northbrook, Illinois. CHI-CHANG CHEN, PhD; CATHERINE B. MCGUINESS, MA, MS; and ROLIN L. WADE, RPh, MS, Health Economics \& Outcomes Research, Real-World Evidence, IQVIA, Plymouth Meeting, Pennsylvania. BRUNO C. MEDEIROS, MD, Department of Medicine, Division of Hematology, Stanford University School of Medicine, Stanford, California.

AUTHOR CORRESPONDENCE: Bhavik J. Pandya, PharmD, Health Economics \& Outcomes Research-Oncology, Medical Affairs Americas, Astellas Pharma U.S., 1 Astellas Way, Northbrook, IL 60062. Tel.: 224.205.5400; E-mail: Bhavik.pandya@astellas.com. 


\section{DISCLOSURES}

This study was funded by Astellas Pharma. Astellas employees were involved in the study design, interpretation of data, writing of the manuscript, and the decision to submit the manuscript for publication. Pandya and Wilson are employees of Astellas Pharma U.S. Walsh was an employee of Astellas Pharma U.S. while the study was conducted. Chen, McGuiness, and Wade are employees of IQVIA, which received funding from Astellas Pharma U.S. Madeiros was employed at Stanford University while this study was conducted and received a consulting fee from Astellas for work on this study.

Data discussed in this study were previously presented at the 59th Annual American Society for Hematology Meeting \& Exposition, 2017; December 9-12, 2017; Atlanta, GA.

\section{ACKNOWLEDGMENTS}

Writing and editorial assistance under the authors' guidance was provided by Patrick Tucker, PhD; Regina Switzer, PhD; and Elizabeth Hermans, PhD, of OPEN Health Communications (Chicago, IL) and was funded by the study sponsor.

\section{DATA-SHARING STATEMENT}

Researchers may request access to anonymized participant level data, trial level data, and protocols from Astellas-sponsored clinical trials at www.clinicalstudydatarequest.com.

For the Astellas criteria on data sharing, see https://clinicalstudydatarequest.com/Study-Sponsors/Study-Sponsors-Astellas.aspx.

\section{REFERENCES}

1. Siegel RL, Miller KD, Jemal A. Cancer statistics, 2019. CA Cancer J Clin. 2019;69(1):7-34.

2. National Cancer Institute. Surveillance, Epidemiology, and End Results Program. Cancer stat facts: leukemia-acute myeloid leukemia (AML). 2016. Available at: https://seer.cancer.gov/statfacts/html/amyl.html. Accessed March 22, 2020.

3. Breems DA, Van Putten WL, Huijgens PC, et al. Prognostic index for adult patients with acute myeloid leukemia in first relapse. J Clin Oncol. 2005;23(9):1969-78
4. Ganzel C, Sun Z, Cripe LD, et al. Very poor long-term survival in past and more recent studies for relapsed AML patients: The ECOG-ACRIN experience. Am J Hematol. 2018;93(8):1074-81. Available at: https://onlinelibrary. wiley.com/doi/full/10.1002/ajh.25162. Accessed April 1, 2020.

5. Hagiwara M, Sharma A, Chung KC, Delea TE. Burden of acute myeloid leukemia (AML) in a U.S. commercially insured population. J Clin Oncol. 2017;35(15 Suppl):e18330.

6. Bryant AL, Deal AM, Walton A, Wood WA, Muss H, Mayer DK. Use of ED and hospital services for patients with acute leukemia after induction therapy: one year follow-up. Leuk Res. 2015;39(4):406-10.

7. Preussler JM, Meyer CL, Mau LW, et al. Healthcare costs and utilization for patients age 50 to 64 years with acute myeloid leukemia treated with chemotherapy or with chemotherapy and allogeneic hematopoietic cell transplantation. Biol Blood Marrow Transplant. 2017;23(6):1021-28.

8. Stein EM, Bonifacio G, Latremouille-Viau D, et al. Treatment patterns, healthcare resource utilization, and costs in patients with acute myeloid leukemia in commercially insured and Medicare populations. J Med Econ. 2018;21(6):556-63.

9. Mahmoud D, Skikne B, Kucmin-Bemelmans I, Alleman C, Hensen M. Overall economic burden of total treatment costs in acute myeloid leukemia throughout the course of the disease [abstract]. Blood. 2012;120(21):3614 Available at: https://ashpublications.org/blood/article/120/21/3614/86271/ Overall-Economic-Burden-of-Total-Treatment-Costs. Accessed April 1, 2020.

10. Meyers J, Yu Y, Kaye JA, Davis KL. Medicare fee-for-service enrollees with primary acute myeloid leukemia: an analysis of treatment patterns, survival, and healthcare resource utilization and costs. Appl Health Econ Health Policy. 2013;11(3):275-86.

11. Shaw LJ, Goyal A, Mehta C, et al. 10-year resource utilization and costs for cardiovascular care. J Am Coll Cardiol. 2018;71(10):1078-89.

12. Pandya BJ, Chen CC, Medeiros BC, et al. Economic and clinical burden of relapsed and/or refractory active treatment episodes in patients with acute myeloid leukemia (AML) in the USA: a retrospective analysis of a commercial payer database. Adv Ther. 2019;36(8):1922-35.

13. Broder MS, Quock TP, Chang E, et al. The cost of hematopoietic stem-cell transplantation in the United States. Am Health Drug Benefits. 2017;10(7):366-74. 
Economic and Clinical Burden of Acute Myeloid Leukemia Episodes of Care in the United States:

A Retrospective Analysis of a Commercial Payer Database

APPENDIX A Study Design and Time Frame

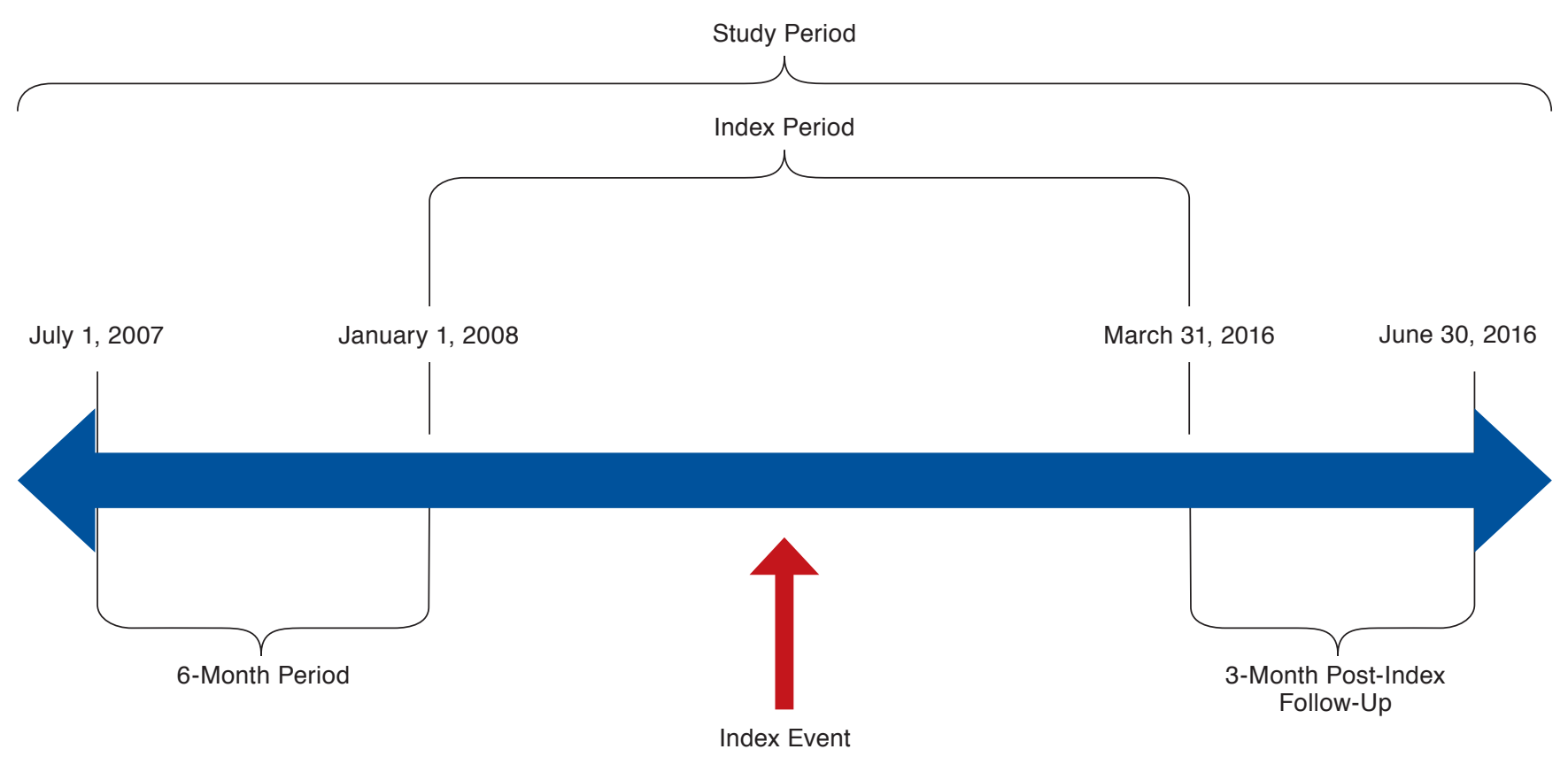

\section{Pre-Index/Baseline Measures \\ Demographics \\ Comorbidies}

Pre-index HRU and costs 


\section{Economic and Clinical Burden of Acute Myeloid Leukemia Episodes of Care in the United States: \\ A Retrospective Analysis of a Commercial Payer Database}

\section{APPENDIX B Overview of Symptom and Toxicity Events}

Adverse events were grouped into the following categories:

Blood and lymphatic system disorders

- Diagnostic proxies:

o Anemia

o Leukopenia

o Neutropenia

o Thrombocytopenia

- Treatment proxies:

o Erythropoietin

o Granulocyte colony-stimulating factor (G-CSF), granulocyte-macrophage CSF

o Blood product administration

Gastrointestinal disorders

- Diagnostic proxies:

o Constipation

o Diarrhea

o Nausea and vomiting

o Decreased appetite and weight loss

o Mucositis

- Treatment proxies:

o Laxatives, anti-diarrheals

o Antiemetics

o Androgenic hormones

Bleeding events

- Diagnostic proxies:

o Intracranial hemorrhage

o Pulmonary hemorrhage

o Gastrointestinal hemorrhage

- Treatment proxies:

o Packed red cells, whole blood

o Surgical intervention

Infections and infestations

- Diagnostic proxies:

o Cellulitis

o Pneumonia

o Pyelonephritis

o Bacteremia/sepsis

o Urinary tract infection

o Candidiasis

o Empyema

o Cytomegalovirus

o Mucormycosis

o Aspergillus

- Treatment proxies:

o Broad-spectrum antibiotics

o Systemic antifungals
Adverse events were grouped into the following categories:

Nervous system disorders

- Diagnostic proxies:

o Dizziness

o Headache

o Mental confusion

o Cerebellar toxicity, ataxia (in combination with, for example, adverse event of antineoplastic drugs)

o Keratoconjunctivitis, Sicca syndrome

o Palmar plantar erythrodysesthesia

o Anxiety, depression, insomnia

- Treatment proxies:

o Tricyclic antidepressants (only with diagnosis of dizziness, headache, ataxia)

Skin and subcutaneous tissue disorders

- Diagnostic proxy: Rash

Vascular disorders

- Diagnostic proxies:

o Hypotension

o Treatment proxies: pressor agents

Renal disorders

- Diagnostic proxies:

o Renal insufficiency

- Treatment proxies:

o Diuretics

o Angiotensin-converting enzyme inhibitors (ACEIs), angiotensin-receptor blockers (ARBs)

Liver disorders

- Diagnostic proxies:

o Hepatotoxicity, nonalcoholic and nonviral cirrhosis

Cardiovascular disorders

- Diagnostic proxies:

o Arrhythmia (excluding QT prolongation)

o Congestive heart failure

- Treatment proxies:

o Antiarrhythmics

o ACEIs, ARBs, beta blockers, digoxin 